\title{
MINIMUM ENERGY CONTROL OF POSITIVE CONTINUOUS-TIME LINEAR SYSTEMS WITH BOUNDED INPUTS
}

\author{
TADEUSZ KACZOREK \\ Faculty of Electrical Engineering \\ Białystok Technical University, ul. Wiejska 45D, 15-351 Białystok, Poland \\ e-mail:kaczorek@isep.pw.edu.pl
}

\begin{abstract}
The minimum energy control problem for positive continuous-time linear systems with bounded inputs is formulated and solved. Sufficient conditions for the existence of a solution to the problem are established. A procedure for solving the problem is proposed and illustrated with a numerical example.
\end{abstract}

Keywords: positive system, continuous time, minimum energy control, bounded inputs.

\section{Introduction}

A dynamical system is called positive if its trajectory starting from any nonnegative initial state remains forever in the positive orthant for all nonnegative inputs. An overview of the state of the art in positive system theory is given by Farina and Rinaldi (2000) as well as Kaczorek (2001). A variety of models having positive behavior can be found in engineering, economics, social sciences, biology and medicine, etc.

Positive fractional linear systems were investigated by Kaczorek (2008a; 2011b; 2011c; 2012). The stability of fractional linear $1 \mathrm{D}$ discrete-time and continuous-time systems was investigated by Busłowicz (2008), Dzieliński and Sierociuk (2008a) as well as Kaczorek (2012), and that of 2D fractional positive linear systems by Kaczorek (2009). The notion of the practical stability of positive fractional discrete-time linear systems was introduced by Kaczorek (2008b). The minimum energy control problem for standard linear systems was formulated and solved by Klamka (1991; 1983; 1976; 2010; 1993; 1977), while for 2D linear systems with variable coefficients by Kaczorek and Klamka (1986). The controllability and minimum energy control problems of fractional discrete-time linear systems were investigated by Klamka (2010; 1993). The minimum energy control of fractional positive continuous-time linear systems was addressed by Kaczorek (2013a), and for descriptor positive discrete-time linear systems by the same author (Kaczorek, 2013b).

In this paper the minimum energy control problem for positive continuous-time linear systems with bounded inputs will be formulated and solved.

The paper is organized as follows. In Section 2 basic definitions and theorems of positive continuous-time linear systems are recalled and necessary and sufficient conditions for the reachability of positive systems are given. The minimum energy control problem of positive linear systems with bounded inputs is formulated and solved in Section 3. Sufficient conditions for the existence of a problem solution are established and a procedure for computation of the optimal inputs and the minimum value of the performance index are also presented. Concluding remarks are given in Section 4.

The following notation will be used: $\mathbb{R}$ is the set of real numbers, $\mathbb{R}^{n \times m}$ is the set of $n \times m$ real matrices, $\mathbb{R}_{+}^{n \times m}$ is the set of $n \times m$ matrices with nonnegative entries and $\mathbb{R}_{+}^{n}=\mathbb{R}_{+}^{n \times 1}, M_{n}$ is the set of $n \times n$ Metzler matrices (real matrices with nonnegative off-diagonal entries), $I_{n}$ is the $n \times n$ identity matrix.

\section{Reachability of positive continuous-time linear systems}

Consider the continuous-time linear system

$$
\dot{x}(t)=A x(t)+B u(t),
$$

where $x(t) \in \mathbb{R}^{n}$ and $u(t) \in \mathbb{R}^{m}$ are the state and input vectors, respectively, and $A \in \mathbb{R}^{n \times n}, B \in \mathbb{R}^{n \times m}$. 
The solution of Eqn. (1) has the form

$$
x(t)=e^{A t} x_{0}+\int_{0}^{t} e^{A(t-\tau)} B u(\tau) \mathrm{d} \tau, \quad x(0)=x_{0} .
$$

Definition 1. (Kaczorek, 2001) The system (1) is called internally positive if and only if $x(t) \in \mathbb{R}_{+}^{n}, t \geq 0$, for any initial conditions $x_{0} \in \mathbb{R}_{+}^{n}$ and all inputs $u(t) \in \mathbb{R}_{+}^{m}$, $t \geq 0$.

Theorem 1. (Kaczorek, 2001) The system (1) is internally positive if and only if

$$
A \in M_{n}, \quad B \in \mathbb{R}_{+}^{n \times m},
$$

where $M_{n}$ is the set of $n \times n$ Metzler matrices.

Definition 2. The positive system (1) (or the positive pair $(A, B))$ is called reachable in time $t \in\left[0, t_{f}\right]$ if for any given final state $x_{f} \in \mathbb{R}_{+}^{n}$ there exists an input $u(t) \in \mathbb{R}_{+}^{m}$, for $t \in\left[0, t_{f}\right]$, that steers the state of the system from the zero initial state $x(0)=0$ to the state $x_{f}$, i.e., $x\left(t_{f}\right)=x_{f}$.

A real square matrix is called monomial if each of its rows and each of its columns contains only one positive entry and the remaining entries are zero.

Theorem 2. The positive system (1) is reachable in time $t \in\left[0, t_{f}\right]$ if and only if the matrix $A \in M_{n}$ is diagonal and the matrix $B \in \mathbb{R}_{+}^{n \times m}$ is monomial.

The proof is similar to that given by Kaczorek (2013a).

\section{Minimum energy control problem for positive systems with bounded inputs}

3.1. Problem formulation. Consider the positive system (1) with $A \in M_{n}$ and monomial $B \in \mathbb{R}_{+}^{n \times n}$. If the system is reachable in time $t \in\left[0, t_{f}\right]$, then there usually exist many different inputs $u(t) \in \mathbb{R}_{+}^{n}$ that steer the system state from $x_{0}=0$ to $x_{f} \in \mathbb{R}_{+}^{n}$. Among these inputs we are looking for an input $u(t) \in \mathbb{R}_{+}^{n}$ satisfying the condition

$$
u(t)<U \in \mathbb{R}_{+}^{n} \quad \text { for } \quad t \in\left[0, t_{f}\right]
$$

that minimizes the performance index

$$
I(u)=\int_{0}^{t_{f}} u^{T}(\tau) Q u(\tau) \mathrm{d} \tau,
$$

where $Q \in \mathbb{R}_{+}^{n \times n}$ is a symmetric positive-definite matrix and $Q^{-1} \in \mathbb{R}_{+}^{n \times n}$.

The minimum energy control problem for the positive continuous-time linear systems (1) with bounded inputs can be stated as follows. Given matrices $A \in M_{n}$, $B \in \mathbb{R}_{+}^{n \times n}, U \in \mathbb{R}_{+}^{n}$ and $Q \in \mathbb{R}_{+}^{n \times n}$ of the performance index (5), $x_{f} \in \mathbb{R}_{+}^{n}$ and $t_{f}>0$, find an input $u(t) \in \mathbb{R}_{+}^{n}$ for $t \in\left[0, t_{f}\right]$ satisfying (4) that steers the system state vector from $x_{0}=0$ to $x_{f} \in \mathbb{R}_{+}^{n}$ while minimizing the performance index (5).
3.2. Problem solution. To solve the problem, we define the matrix

$$
\begin{aligned}
W & =W\left(t_{f}, Q\right) \\
& =\int_{0}^{t_{f}} e^{A\left(t_{f}-\tau\right)} B Q^{-1} B^{T} e^{A^{T}\left(t_{f}-\tau\right)} \mathrm{d} \tau .
\end{aligned}
$$

From (6) and Theorem 2.2 it follows that the matrix (6) is monomial if and only if the fractional positive system (1) is reachable in time $\left[0, t_{f}\right]$. In this case we may define the input

$$
\hat{u}(t)=Q^{-1} B^{T} e^{A^{T}\left(t_{f}-t\right)} W^{-1} x_{f} \quad \text { for } \quad t \in\left[0, t_{f}\right] .
$$

Note that the input (7) satisfies the condition $u(t) \in$ $\mathbb{R}_{+}^{n}$ for $t \in\left[0, t_{f}\right]$ if

$$
Q^{-1} \in \mathbb{R}_{+}^{n \times n} \quad \text { and } \quad W^{-1} x_{f} \in \mathbb{R}_{+}^{n} .
$$

Theorem 3. Let the positive system (1) be reachable in time $\left[0, t_{f}\right]$ and let $\bar{u}(t) \in \mathbb{R}_{+}^{n}$ for $t \in\left[0, t_{f}\right]$ be an input that steers the state of the positive system (1) from $x_{0}=$ 0 to $x_{f} \in \mathbb{R}_{+}^{n}$ and satisfies the condition (4). Then the input (7) also steers the system state from $x_{0}=0$ to $x_{f} \in$ $\mathbb{R}_{+}^{n}$ and minimizes the performance index (5), i.e., $I(\hat{u}) \leq$ $I(\bar{u})$.

The minimal value of the performance index (5) is equal to

$$
I(\hat{u})=x_{f}^{T} W^{-1} x_{f}
$$

Proof. If the conditions (8) are met, then the input (7) is well defined and $\hat{u}(t) \in \mathbb{R}_{+}^{n}$ for $t \in\left[0, t_{f}\right]$. We shall show that the input steers the system state from $x_{0}=0$ to $x_{f} \in$ $\mathbb{R}_{+}^{n}$. Substitution of (7) into (2) for $t=t_{f}$ and $x_{0}=0$ yields

$$
\begin{aligned}
& x\left(t_{f}\right) \\
& =\int_{0}^{t_{f}} e^{A\left(t_{f}-\tau\right)} B \hat{u}(\tau) \mathrm{d} \tau \\
& =\int_{0}^{t_{f}} e^{A\left(t_{f}-\tau\right)} B Q^{-1} B^{T} e^{A^{T}\left(t_{f}-\tau\right)} \mathrm{d} \tau W^{-1} x_{f} \\
& =x_{f}
\end{aligned}
$$

since (6) holds. By assumption, the inputs $\bar{u}(t)$ and $\hat{u}(t)$, $t \in\left[0, t_{f}\right]$, steer the system state from $x_{0}=0$ to $x_{f} \in \mathbb{R}_{+}^{n}$. Hence

$$
\begin{aligned}
x_{f} & =\int_{0}^{t_{f}} e^{A\left(t_{f}-\tau\right)} B \bar{u}(\tau) \mathrm{d} \tau \\
& =\int_{0}^{t_{f}} e^{A\left(t_{f}-\tau\right)} B \hat{u}(\tau) \mathrm{d} \tau
\end{aligned}
$$

or

$$
\int_{0}^{t_{f}} e^{A\left(t_{f}-\tau\right)} B[\bar{u}(\tau)-\hat{u}(\tau)] \mathrm{d} \tau=0
$$


By transposition of (11b) and postmultiplication by $W^{-1} x_{f}$, we obtain

$$
\int_{0}^{t_{f}}[\bar{u}(\tau)-\hat{u}(\tau)]^{T} B^{T} e^{A^{T}\left(t_{f}-\tau\right)} \mathrm{d} \tau W^{-1} x_{f}=0 .
$$

Substitution of (7) into (12) yields

$$
\begin{aligned}
\int_{0}^{t_{f}}[\bar{u}(\tau) & -\hat{u}(\tau)]^{T} B^{T} e^{A^{T}\left(t_{f}-\tau\right)} \mathrm{d} \tau W^{-1} x_{f} \\
= & \int_{0}^{t_{f}}[\bar{u}(\tau)-\hat{u}(\tau)]^{T} Q \hat{u}(\tau) \mathrm{d} \tau=0 .
\end{aligned}
$$

Using (13), it is easy to verify that

$$
\begin{aligned}
& \int_{0}^{t_{f}} \bar{u}(\tau)^{T} Q \bar{u}(\tau) \mathrm{d} \tau \\
& =\int_{0}^{t_{f}} \hat{u}(\tau)^{T} Q \hat{u}(\tau) \mathrm{d} \tau \\
& \quad+\int_{0}^{t_{f}}[\bar{u}(\tau)-\hat{u}(\tau)]^{T} Q[\bar{u}(\tau)-\hat{u}(\tau)] \mathrm{d} \tau .
\end{aligned}
$$

From (14) it follows that $I(\hat{u})<I(\bar{u})$ since the second term on the right-hand side of the inequality is nonnegative.

To find the minimal value of the performance index (5), we substitute (7) into (5) and obtain

$$
\begin{aligned}
I(\hat{u})= & \int_{0}^{t_{f}} \hat{u}^{T}(\tau) Q \hat{u}(\tau) \mathrm{d} \tau \\
= & x_{f}^{T} W^{-1} \int_{0}^{t_{f}} e^{A\left(t_{f}-\tau\right)} B Q^{-1} B^{T} e^{A^{T}\left(t_{f}-\tau\right)} \mathrm{d} \tau \\
& \times W^{-1} x_{f} \\
= & x_{f}^{T} W^{-1} x_{f}
\end{aligned}
$$

since (6) holds.

From (7) we have

$$
\frac{\mathrm{d} \hat{u}(t)}{\mathrm{d} t}=-E A^{T} e^{A^{T}\left(t_{f}-t\right)} F,
$$

where

$$
E=Q^{-1} B^{T}, \quad F=W^{-1} x_{f} .
$$

Using (17) we may find $t \in\left[0, t_{f}\right]$ for which $\hat{u}(t) \in \mathbb{R}_{+}^{n}$ reaches its maximal value. Note that if all the eigenvalues of the matrix $A$ have positive real parts, then $\hat{u}(t)$ reaches its maximal value for $t=0$, and if they have negative real parts, then this value is attained for $t=t_{f}$.

From the above we have the following procedure for computation of the optimal inputs satisfying the condition (4) that steer the state of the system from $x_{0}=0$ to $x_{f} \in$ $\mathbb{R}_{+}^{n}$ and minimize the performance index (5).

\section{Procedure 1.}

Step 1. Knowing $A \in M_{n}$, compute $e^{A t}$.
Step 2. Using (6), compute the matrix $W$ knowing the matrices $A, B, Q$ for some $t_{f}$.

Step 3. Using (7) and (16), compute the input (7) and $t_{f}$ satisfying the condition (4) for given $U \in \mathbb{R}_{+}^{n}$ and $x_{f} \in$ $\mathbb{R}_{+}^{n}$.

Step 4. Using (9), compute the minimal value of the performance index.

Example 1. Consider the positive system (1) with matrices

$$
\begin{aligned}
A=\left[\begin{array}{cc}
a_{1} & 0 \\
0 & a_{2}
\end{array}\right], \quad B=\left[\begin{array}{cc}
0 & b_{1} \\
b_{2} & 0
\end{array}\right], \\
a_{k}>0, \quad b_{k}>0, \quad k=1,2
\end{aligned}
$$

and the performance index (5) with

$$
Q=\left[\begin{array}{cc}
q_{1} & 0 \\
0 & q_{2}
\end{array}\right], \quad q_{k}>0, k=1,2
$$

Compute the bounded input $\hat{u}(t)$ satisfying

$$
\hat{u}(t)=\left[\begin{array}{c}
\hat{u}_{1}(t) \\
\hat{u}_{2}(t)
\end{array}\right]<\left[\begin{array}{c}
U_{1} \\
U_{2}
\end{array}\right]
$$

for $t \in\left[0, t_{f}\right]$ that steers the state of the system from zero state to $x_{f}=\left[\begin{array}{ll}x_{f 1} & x_{f 2}\end{array}\right]^{T} \in \mathbb{R}_{+}^{2}$ ( $T$ denotes the transpose) and minimizes the performance index.

Using Procedure 1, we obtain the following.

Step 1. In this case, we have

$$
e^{A t}=\left[\begin{array}{cc}
e^{a_{1} t} & 0 \\
0 & e^{a_{2} t}
\end{array}\right]
$$

Step 2. Using (18), 19) and (20), we obtain

$$
\begin{aligned}
W & =\int_{0}^{t_{f}} e^{A\left(t_{f}-\tau\right)} B Q^{-1} B^{T} e^{A^{T}\left(t_{f}-\tau\right)} \mathrm{d} \tau \\
& =\int_{0}^{t_{f}} e^{A \tau} B Q^{-1} B^{T} e^{A^{T} \tau} \mathrm{d} \tau \\
& =\int_{0}^{t_{f}}\left[\begin{array}{cc}
b_{1}^{2} q_{2}^{-1} e^{2 a_{1} \tau} & 0 \\
0 & b_{2}^{2} q_{1}^{-1} e^{2 a_{2} \tau}
\end{array}\right] \mathrm{d} \tau \\
& =\left[\begin{array}{cc}
\frac{b_{1}^{2} q_{2}^{-1}}{2 a_{1}}\left(e^{2 a_{1} t_{f}}-1\right) & 0 \\
0 & \frac{b_{2}^{2} q_{1}^{-1}}{2 a_{2}}\left(e^{2 a_{2} t_{f}}-1\right)
\end{array}\right] .
\end{aligned}
$$


Step 3. Using (7), (18), (19) and (21), we obtain

$$
\begin{aligned}
\hat{u}(t) & Q^{-1} B^{T} e^{A^{T}\left(t_{f}-t\right)} W^{-1} x_{f} \\
= & {\left[\begin{array}{cc}
q_{1}^{-1} & 0 \\
0 & q_{2}^{-1}
\end{array}\right]\left[\begin{array}{cc}
0 & b_{1} \\
b_{2} & 0
\end{array}\right] } \\
& \times\left[\begin{array}{cc}
e^{a_{1}\left(t_{f}-t\right)} & 0 \\
0 & e^{a_{2}\left(t_{f}-t\right)}
\end{array}\right] \\
& \times\left[\begin{array}{cc}
\frac{b_{1}^{2} q_{2}^{-1}}{2 a_{1}}\left(e^{2 a_{1} t_{f}}-1\right) & \frac{b_{2}^{2} q_{1}^{-1}}{2 a_{2}}\left(e^{2 a_{2} t_{f}}-1\right)
\end{array}\right] \\
& \times\left[\begin{array}{c}
x_{f 1} \\
x_{f 2}
\end{array}\right] \\
= & {\left[\begin{array}{l}
\frac{2 a_{2}}{b_{2}} e^{a_{2}\left(t_{f}-t\right)}\left(e^{2 a_{2} t_{f}}-1\right)^{-1} x_{f 2} \\
\frac{2 a_{1}}{b_{1}} e^{a_{1}\left(t_{f}-t\right)}\left(e^{2 a_{1} t_{f}}-1\right)^{-1} x_{f 1}
\end{array}\right] . }
\end{aligned}
$$

The minimal value of $t_{f}$ satisfying the condition (4) can be found from the inequality

$$
\begin{gathered}
{\left[\begin{array}{c}
\frac{2 a_{2}}{b_{2}} e^{a_{2}\left(t_{f}-t\right)}\left(e^{2 a_{2} t_{f}}-1\right)^{-1} x_{f 2} \\
\frac{2 a_{1}}{b_{1}} e^{a_{1}\left(t_{f}-t\right)}\left(e^{2 a_{1} t_{f}}-1\right)^{-1} x_{f 1}
\end{array}\right]} \\
<\left[\begin{array}{c}
U_{1} \\
U_{2}
\end{array}\right] \text { for } t \in\left[0, t_{f}\right] .
\end{gathered}
$$

From (23), we have

$$
\begin{gathered}
e^{2 a_{2} t_{f}}-\frac{2 a_{2} x_{f 2}}{b_{2} U_{1}} e^{a_{2} t_{f}}-1>0, \\
e^{2 a_{1} t_{f}}-\frac{2 a_{1} x_{f 1}}{b_{1} U_{2}} e^{a_{1} t_{f}}-1>0 .
\end{gathered}
$$

Solving the inequalities 24 with respect to $t_{f}$, we obtain

$$
\begin{aligned}
& t_{f}>\frac{1}{a_{2}} \ln \left[\frac{a_{2} x_{f 2}}{b_{2} U_{1}}+\sqrt{\left(\frac{a_{2} x_{f 2}}{b_{2} U_{1}}\right)^{2}+1}\right], \\
& t_{f}>\frac{1}{a_{1}} \ln \left[\frac{a_{1} x_{f 1}}{b_{1} U_{2}}+\sqrt{\left(\frac{a_{1} x_{f 1}}{b_{1} U_{2}}\right)^{2}+1}\right]
\end{aligned}
$$

and

$$
\begin{aligned}
t_{f}= & \max \left\{\frac{1}{a_{2}} \ln \left[\frac{a_{2} x_{f 2}}{b_{2} U_{1}}+\sqrt{\left(\frac{a_{2} x_{f 2}}{b_{2} U_{1}}\right)^{2}+1}\right]\right. \\
& \left.\frac{1}{a_{1}} \ln \left[\frac{a_{1} x_{f 1}}{b_{1} U_{2}}+\sqrt{\left(\frac{a_{1} x_{f 1}}{b_{1} U_{2}}\right)^{2}+1}\right]\right\}
\end{aligned}
$$

For example, for $a_{1}=2, a_{2}=3, b_{1}=b_{2}=1, U_{1}=$ $U_{2}=1$ and $x_{f}=\left[\begin{array}{ll}1 & 1\end{array}\right]^{T}$, from (22) we obtain $\hat{u}_{1}(t)$ and $\hat{u}_{2}(t)$ for $t \in[0,1]$ shown in Fig. 1 .

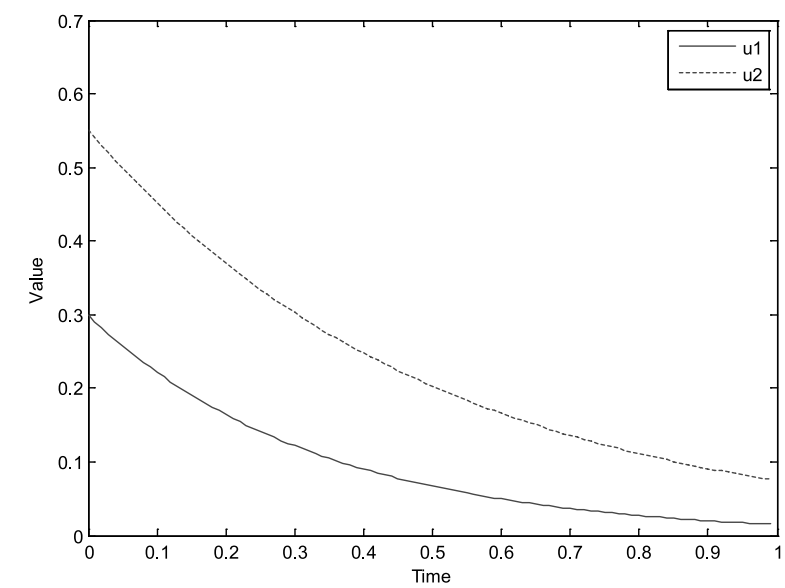

Fig. 1. Values of optimal input at time $t \in[0,1]$.

Note that $\hat{u}_{1}(t)$ and $\hat{u}_{2}(t)$ reach their maximum values for $t=0$ since the eigenvalues $a_{1}, a_{2}$ of $A$ are positive.

From (24) for the same data we obtain

$$
\begin{gathered}
\frac{1}{a_{2}} \ln \left[\frac{a_{2} x_{f 2}}{b_{2} U_{1}}+\sqrt{\left(\frac{a_{2} x_{f 2}}{b_{2} U_{1}}\right)^{2}+1}\right] \\
=\frac{1}{3} \ln [3+\sqrt{10}]=0.6061, \\
\frac{1}{a_{1}} \ln \left[\frac{a_{1} x_{f 1}}{b_{1} U_{2}}+\sqrt{\left(\frac{a_{1} x_{f 1}}{b_{1} U_{2}}\right)^{2}+1}\right] \\
=\frac{1}{2} \ln [2+\sqrt{5}]=0.7218
\end{gathered}
$$

and

$$
t_{f}=\max \{0.6061,0.7218\}=0.7218 .
$$

Step 4. The minimal value of the performance index (9) is equal to

$$
\begin{aligned}
& I(\hat{u}) \\
& =x_{f}^{T} W^{-1} x_{f}=\left[\begin{array}{ll}
x_{f 1} & x_{f 2}
\end{array}\right] \\
& \quad \times\left[\begin{array}{cc}
\frac{b_{1}^{2} q_{2}^{-1}}{2 a_{1}}\left(e^{2 a_{1} t_{f}}-1\right) & 0 \\
0 & \frac{b_{2}^{2} q_{1}^{-1}}{2 a_{2}}\left(e^{2 a_{2} t_{f}}-1\right)
\end{array}\right] \\
& \quad \times\left[\begin{array}{c}
x_{f 1} \\
x_{f 2}
\end{array}\right] \\
& =\frac{2 a_{1} q_{2}}{b_{1}^{2}}\left(e^{2 a_{1} t_{f}}-1\right)^{-1} x_{f 1}^{2} \\
& +\frac{2 a_{2} q_{1}}{b_{2}^{2}}\left(e^{2 a_{2} t_{f}}-1\right)^{-1} x_{f 2}^{2} .
\end{aligned}
$$




\section{Concluding remarks}

Necessary and sufficient conditions for the reachability of positive continuous-time linear systems were established (Theorem 2). The minimum energy control problem for positive continuous-time linear systems with bounded inputs was formulated and solved. Sufficient conditions for the existence of a solution to the problem were given (Theorem 3), and a procedure for computation of optimal input satisfying the condition (4) and the minimal value of the performance index was proposed. The effectiveness of the procedure was demonstrated on a numerical example. The presented method can be extended to positive discrete-time linear systems as well as fractional positive continuous-time and discrete-time linear systems with bounded inputs.

\section{Acknowledgment}

This work was supported by the Białystok Technical University under a grant no. S/WE/1/11.

\section{References}

Busłowicz M. (2008). Stability of linear continuous time fractional order systems with delays of the retarded type, Bulletin of the Polish Academy of Sciences: Technical Sciences 56(4): 319-324.

Dzieliński A., Sierociuk D. and Sarwas G. (2009). Ultracapacitor parameters identification based on fractional order model, Proceedings of ECC'09, Budapest, Hungary.

Dzieliński A. and Sierociuk D. (2008a). Stability of discrete fractional order state-space systems, Journal of Vibrations and Control 14(9/10): 1543-1556.

Farina L. and Rinaldi S. (2000). Positive Linear Systems: Theory and Applications, J. Wiley, New York, NY.

Kaczorek T. (1992). Linear Control Systems, Research Studies Press/J.Wiley, New York, NY.

Kaczorek T. (2001). Positive $1 D$ and $2 D$ Systems, Springer-Verlag, London.

Kaczorek T. (2008a). Fractional positive continuous-time systems and their reachability, International Journal of Applied Mathematics Computer Science 18(2): 223-228, DOI: 10.2478/v10006-008-0020-0.

Kaczorek T. (2008b) Practical stability of positive fractional discrete-time linear systems, Bulletin of the Polish Academy of Sciences: Technical Sciences 56(4): 313-317.

Kaczorek T. (2008c). Reachability and controllability to zero tests for standard and positive fractional discrete-time systems, Journal Européen des Systèmes Automatisés 42(6-8): 769-787.

Kaczorek T. (2009). Asymptotic stability of positive fractional 2D linear systems, Bulletin of the Polish Academy of Sciences: Technical Sciences 57(3): 289-292.

Kaczorek T. (2011a) Controllability and observability of linear electrical circuits, Electrical Review 87(9a): 248-254.
Kaczorek T. (2011b). Positivity and reachability of fractional electrical circuits, Acta Mechanica et Automatica 5(2): $42-51$.

Kaczorek T. (2011c). Positive linear systems consisting of $n$ subsystems with different fractional orders, Transactions of IEEE: Circuits and Systems 58(6): 1203-1210.

Kaczorek T. (2011d) Checking of the positivity of descriptor linear systems by the use of the shuffle algorithm, Archive of Control Sciences 21(3): 287-298.

Kaczorek T. (2012). Selected Problems of Fractional Systems Theory, Springer-Verlag, Berlin.

Kaczorek T. (2013a) Minimum energy control of fractional positive continuous-time linear systems, Proceedings of the 18th International Conference on Methods and Models in Automation and Robotics, Międzyzdroje, Poland, NN 514638940.

Kaczorek T. (2013b). Minimum Energy Control of Descriptor Positive Discrete-time Linear Systems, Compel, (in press).

Kaczorek T. and Klamka J. (1986). Minimum energy control of 2D linear systems with variable coefficients, International Journal of Control 44(3): 645-650.

Klamka J. (1991) Controllability of Dynamical Systems, Kluwer Academic Press, Dordrecht.

Klamka J. (1983). Minimum energy control of 2D systems in Hilbert spaces, System Sciences 9(1-2): 33-42.

Klamka J. (1976). Relative controllability and minimum energy control of linear systems with distributed delays in control, IEEE Transactions on Automatic Control 21(4): 594-595.

Klamka J. (2010). Controllability and minimum energy control problem of fractional discrete-time systems, in $\mathrm{D}$. Baleanu, Z.B. Guvenc and J.A. Tenreiro Machado (Eds.), New Trends in Nanotechology and Fractional Calculus, Springer-Verlag, New York, NY, pp. 503-509.

Klamka J. (1993). Controllability of dynamical systems-a survey, Archives of Control Sciences 2(3-4): 281-307.

Klamka J. (1977). Minimum energy control of discrete systems with delays in control, International Journal of Control 26(5): 737-744.

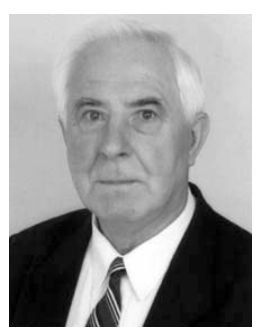

Tadeusz Kaczorek received the M.Sc., Ph.D and D.Sc. degrees in electrical engineering from the Warsaw University of Technology in 1956, 1962 and 1964, respectively. In the years 1968-69 he was the dean of the Electrical Engineering Faculty, and in the period of 1970-73 he was a deputy rector of the Warsaw University of Technology. In 1971 he became a professor and in 1974 a full professor at the same university. Since 2003 he has been a professor at Białystok Technical University. In 1986 he was elected a corresponding member and in 1996 a full member of the Polish Academy of Sciences. In the years 1988-1991 he was the director of the Research Centre of the Polish Academy of Sciences in Rome. In 2004 he was elected an honorary member of the Hungarian Academy of Sciences. He has been granted honorary doctorates by nine universities. His research interests cover systems theory, especially singular multidimensional systems, positive multidimensional systems, singular positive $1 \mathrm{D}$ and $2 \mathrm{D}$ systems, as well 
as positive fractional $1 \mathrm{D}$ and $2 \mathrm{D}$ systems. He initiated research in the field of singular 2D, positive 2D and positive fractional linear systems. He has published 24 books (six in English) and over 1000 scientific papers. He has also supervised $69 \mathrm{Ph} . \mathrm{D}$. theses. He is the editor-in-chief of the Bulletin of the Polish Academy of Sciences: Technical Sciences and a member of editorial boards of ten international journals.

Received: 25 April 2013

Revised: 27 September 2013 